\title{
HOW THE EYES AFFECT THE I: GAZE PERCEPTION, COGNITION AND THE ROBOT-HUMAN INTERFACE
}

\author{
Stephen R. H. Langton \\ Department of Psychology \\ University of Stirling \\ Stirling, UK \\ E-mailsrhl1@stirling.ac.uk
}

\begin{abstract}
A good deal of research has shown that humans are particularly sensitive to gaze direction. Indeed we may well have evolved neural mechanisms dedicated to the perception of the eyes and eye-gaze direction. As well as providing a very strong signal to our perceptual systems eye-gaze also produces a number of cognitive effects. Here I review a number of studies suggesting that both eye-gaze direction, and head orientation are processed automatically by our cognitive systems interfering with the processing of auditory directional information, triggering reflexive shifts of attention, influencing the information we extract from natural scenes and the performance of certain communicative tasks. Given the potential for social attention cues to influence aspects of cognitive activity, it would seem critical for designers to pay particular attention to the appearance and movement of the eyes and head in the creation of robot-human interfaces.
\end{abstract}

\section{Introduction}

For many, the face is an interesting stimulus to study because of the range of information it communicates. In addition to someone's age, race and gender, we use faces to identify other members of our species, their facial expression help us understand how they are feeling and we use the configuration of their lips, teeth and tongue to aid us in interpreting what they are saying [see Bruce for a review]. Models of face processing have emphasised how many of these meanings are extracted from the face in parallel by functionally independent processing systems [2, 3]. Bruce and Young's influential model [2], for example, includes parallel "routes" for identity, expression and facial speech analysis, a position for which there is now converging empirical support $[4$, but see 5 and 6 for some complications]

However, an additional facial signal - gaze - does not appear in these models and yet it makes a substantial contribution to interpersonal communication. For example, social psychologists have long known that gaze is used in functions such as regulating turn taking in conversation, expressing intimacy and exercising social control [7]. It is only in recent years that cognitive psychologists have added to some early work on the perception of eye gaze, and begun studying some of the cognitive processes underlying the analysis of gaze direction, and how gaze might influence other aspects of information processing. In this paper I briefly discuss some issues in gaze perception and go on to review work suggesting that we may have evolved neural mechanisms for processing where someone is directing their attention, I then go on to describe how these social attention signals can influence information processing at several levels: they can capture visual attention and influence scene perception; they can interfere with both simple directional decisions and more complicated visuo-spatial tasks; and finally - under certain circumstances - they can have a negative impact on children's communication. I conclude by discussing the implications of these findings for the design of the interface for "socially intelligent" robots.

\section{Gaze Perception}

On the face of it, there would appear to be enormous adaptive advantage to being able to detect when you are the object of another's attention, and - if someone isn't looking directly at you - where exactly they are looking. Direct gaze might mean you are about to become a meal, or threatened in some way, so the rapid detection of a direct gaze signal would facilitate any remedial action. The direction of another's gaze might also signal the location of food, a potential predator, or a mate and so rapidly computing their gaze direction and following their line of regard would be a useful ability, allowing more efficient processing of the focus of their attention. Indeed, evolution seems to have furnished us with excellent sensitivity to gaze direction. Studies have shown that with the head pointing directly forward, participants are able to discriminate angles of gaze of from $2.8^{\circ}[8]$ to as little as $0.75^{\circ}$ [9].

So how do we actually compute the line of regard of another person's gaze? The answer would appear to be quite simple: all we need to do is work out where the iris is in the eye. However, this can be done in at least two ways. The first, a spatial computation, involves comparing the location of some feature on the surface of the eyeball, such as the high contrast limbus (the junction between the sclera and the iris), to a fixed feature such as the corner of the eye. However, if this were the method used to compute gaze direction, we might expect people to become less accurate at judging gaze direction as distance from the gazer increases. In contrast to this, at least two studies have shown that this ability remains relatively constant until the limits of visual acuity are reached [10, 11]. Watt [10 see also 12] has therefore suggested a second mechanism. Instead of a spatial 
calculation, this method effectively measures the relative luminance of the visible sclera on either side of the iris. As the eye turns, the relative areas - and hence the relative luminance - of each part of the sclera changes. For instance, as another's eye turns to the left, the luminance of the right hand side of the sclera increases relative to the luminance of the left hand part. Thus, this scleral contrast is a direct function of eye direction and a particularly simple measurement to perform on the eye. In fact, Watt [10] has shown how the output of vertically oriented simple cells from striate cortex can, in principal, signal the direction of gaze using this method. In other words, gaze direction can be recovered from the retinal image of the eye using non-specialised neural machinery. The idea is that the physical structure of the eye may have evolved in such a way as to exploit neurones that already exist for other purposes.

\subsection{A Gaze Module}

However, because of the apparent adaptive advantage of rapid gaze detection, might not evolution have furnished us with some more specialised neural machinery? Is there any evidence for some kind dedicated gaze "module"?

Using single cell recording techniques, Perrett and his group $[13,14]$ have identified cells in the STS region of the macaque temporal lobe which respond maximally to particular gaze directions. For example, one population of cells fires with maximum frequency when the animal sees another individual gazing upwards whilst another population of cells respond to downward directed gaze. Removal of this region renders the macaque unable to make gaze direction judgements but spares other faceprocessing abilities [15]. Neuropsychological work also points toward a functional specialisation for gaze detection. Studies with brain injured human patients have indicated dissociations between gaze perception and face recognition abilities $[15,16]$. More recently, functional neuroimaging studies have confirmed that different cortical regions are active when participants make decisions about gaze direction and identity [17].

Thus, neuroscience has provided us with some good evidence for the existence for some kind of dedicated system for computing gaze direction. Of course, the two viewpoints discussed above are not necessarily mutually exclusive: the eye has probably evolved as an excellent signaler of gaze direction, and perceivers have probably evolved brain mechanisms with which to perceive it.

\subsection{Head Orientation}

The direction in which someone is attending, however, can be signaled by cues other than eye-gaze direction. In fact, many have argued that accurate perception of gaze direction depends on combining information from eye-direction and head orientation $[18$, but see 10 for an alternative perspective]. Whether or not head orientation has to be computed in perceiving gaze direction, it is clearly an important cue in its own right: it can signal the direction of attention when the eyes are obscured by shadow or sunglasses, or when the face is viewed at too great a distance to resolve eye-direction. Like eye-gaze, head orientation can, in principal, be computed fairly early in visual processing using the deviation of the head from bilateral symmetry as a cue [18]. Furthermore, our own research has indicated that head orientation seems to be processed automatically by observers (see below). Studies examining gaze following in children have also highlighted the importance of the head as a salient cue to attention direction. Children will follow their mother's eye-gaze from about 14-18 months of age. However, prior to this, they ignore the eyes and simply use the position of the head as an attention following cue [19].

Finally, Perrett's single cell recording studies also indicate a special role for the head and body posture. The STS cells in the macaques were not simply sensitive to eye-gaze direction, but seemed to respond to conjunctions of eye, head and body position [13, 14]. For instance, those cells that were particularly active when presented with a pair of eyes directed downwards also responded strongly when heads were directed downwards or when the body adopted a quadrupedal posture. Accordingly, Perrett \& Emery [20] postulated the existence of a direction of attention director (DAD) which combines information from separate detectors analysing the direction of the eyes, the head and the body.

In summary, we are remarkably good at detecting gaze direction and this may be because the eye has evolved as an excellent signaling device and because we have evolved specialised neural systems for its processing. However, whilst there is some good evidence for the existence of a gaze module, we might think of this as being one part of a larger system, perhaps also containing a head orientation module, designed to compute the direction of social attention.

\section{Gaze and Cognition}

The material reviewed above clearly suggests that the eyes and the angle of the head act as powerful signals to our perceptual systems. However, some recent work has suggested that gaze direction also has effects on various aspects of cognition. Experiments with normal adult participants have shown that directional information from head and gaze cues is extracted automatically by observers and can influence the processing of spoken directional words. Gaze and head cues also exert effects on viewers' attentional systems, triggering reflexive shifts of attention which may well have implications for the way in which we process information in natural scenes.

\subsection{Interference Effects}

If, as suggested above, gaze direction and head orientation are processed by dedicated modules, we might expect these sources of information to be processed rapidly and manditorily [21]. Experimental evidence in support of this notion comes from a series of recent studies using a Stroop-type interference paradigm 
$[22,23,24]$. In these experiments, the directional cues of interest are placed into conflict and participants are asked to make a directional decision to one source of information whilst ignoring the other. In one study [22], participants were shown the stimuli illustrated in Figure 1 , one at a time on a computer screen. In one block of trials they were asked to press a button on the keyboard contingent on the direction in which the head was oriented. Although participants were asked to ignore the gaze direction, the results indicated that they were unable to do so. Reaction times were faster when the eye-gaze and head were oriented in the same direction than when they were oriented in opposite directions. More surprisingly an identical pattern of results was obtained when participants were asked to do the opposite task, that is respond on the basis of the gaze direction and ignore the orientation of the head. The results of this experiment suggest that participants are unable to ignore directional information provided by the eyes and by the orientation of the head. In other words, directional information is extracted obligatorily from both these stimuli.

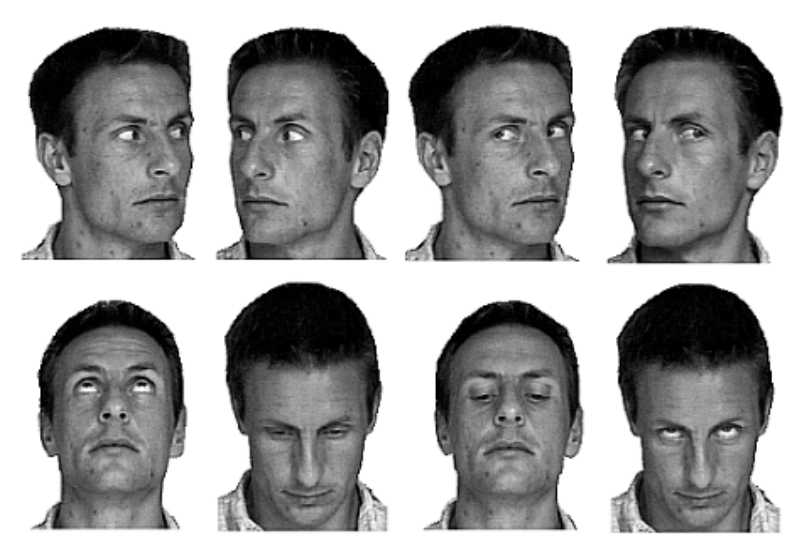

Figure 1. Faces with congruent and incongruent head and gaze orientation .

In a second experiment, participants were again presented with the stimuli in Figure 1, but this time they were asked to ignore these images and to respond to a spoken directional word ("up", "down", "left" or "right") that was presented at the same time as each face. Head and gaze cues were found to exert equal and independent effects on the speed of participants' responses to spoken directional words. So, on hearing the word "up" participants responded faster when they saw the gaze and head oriented upwards compared with trials when the head and gaze were directed downwards. However, this effect was completely eliminated when head and gaze were oriented in opposite directions.

Other similar studies have indicated that, in addition to head and gaze cues, pointing gestures are also processed automatically when participants are asked to make directional decisions to head/gaze direction, to spoken directional words, or even to arrows [23, 24]. Taking all of these studies together, it seems that observers find it hard to ignore directional cues provided by the head, the eyes and pointing gestures, and process this information in parallel. Information from all of these cues is therefore available when a decision has to be made about where another individual is directing their attention, or perhaps when any directional decision is required. Gaze, head and gestural information can exert effects on one another and on the processing of directional information, even if presented in a completely separate modality.

\subsection{Visual Orienting}

Most of us will have experienced the tendency to follow one another's gaze. For example, if - whilst in conversation - you suddenly turn your head to one side, you might find your conversational partner reorienting their gaze in the same direction. Anecdotally then, there appears to be some suggestion that shifts in another's line of regard might trigger shifts of one's own attention. Indeed, developmental psychologists have studied when this gaze-following tendency develops in childhood [25, $26,27]$ as it has been suggested that joint attention behaviours are important milestones in the development of a theory of mind [28]. However, a number of recent studies have suggested that gaze-following might well be more than simply a tendency. This work has indicated that shifts in the direction of another's eye-gaze or head orientation can trigger reflexive shifts in an observer's visual attention [29, 30, 31].

These studies have adopted the cueing paradigm devised by Posner [32]. For example Langton \& Bruce [29] asked participants to press the space bar on a keyboard as soon as they detected a target letter which could appear at one of four locations on a computer monitor. Either $100 \mathrm{~ms}$ or $1000 \mathrm{~ms}$ prior to the appearance of the target, a face appeared in the centre of the screen that was oriented toward one of the possible target locations. Targets could therefore appear in either cued or uncued locations. Participants were told (correctly) that following the appearance of a face, the target letter was equally likely to appear in any of the four possible locations. In other words, the cue was completely uninformative regarding the likely location of the target and therefore should be ignored. However, the results indicated that participants were not able to comply with these instructions. At the shorter, $100 \mathrm{~ms}$ cue-target interval, detection times were faster for targets appearing in cued locations than for those appearing in uncued locations. However, this cueing effect had vanished within $1000 \mathrm{~ms}$ of the presentation of the face cue.

On the basis of this pattern of results, Langton and Bruce concluded that the face cues triggered a kind of reflexive or exogenous shift of visual attention that is normally associated with a change in luminance, or the abrupt onset of a stimulus in the periphery of vision. Identical conclusions were reached by Friesen and Kingstone [30] and Driver et al. [31] and who obtained broadly similar results using eye-gaze direction as their cueing stimuli. 
Thus, once again gaze cues can exert their effects on the processing of other stimuli; in this case the detection of the target stimuli.

\subsection{Scene Perception and Change Blindness}

Social attention signals such as gaze and head orientation clearly, then, exert effects on an observer's attention systems. Some of our recent work further suggests that this relationship might operate in the perception of more natural scenes and may function to help an observer construct the meaning of such a scene. In one study Langton \& O'Donnell [33] used the flicker paradigm, introduced by Rensink et al. [34] to examine whether the presence of someone's gaze cues in a scene might influence an observer's ability to detect a change made to that scene.

In the flicker paradigm, participants are presented with alternating versions of a scene and a modified version of that scene. These modifications might include fairly large changes such as the removal of an object, a change in colour or location of an object, or the substitution of an object with another object. Ordinarily the motion transients produced by these changes make them trivially easy to detect and report. However, if the two versions of the scene are separated by the presentation of a brief blank interval, included to simulate the visual suppression caused by a saccadic eyemovement, observers take a surprisingly long time to spot these changes. This so-called change blindness effect has also been noted when the change to the scene occurs during an actual saccadic eye-movement, a film cut, or when the change occurs at the same time as small local transients such as "mudsplashes" are presented on the display (see [35] for a recent review of change blindness research).

A widely held view in the change blindness literature is that attention is necessary to perceive changes made to the scenes [34, 36, 37, 38]. The idea is that the disappearance or substitution of an object produces a retinal transient that would normally attract the focus of visual attention. The allocation of attention to the object then serves to maintain its representation in visual short term memory so that a comparison can be made when a transformation to that object subsequently occurs. However, an intervening flicker, mudsplash, film cut or eye blink serves to swamp the motion signals normally associated with an object change, thus preventing attention from being automatically deployed toward this region. The object representation will therefore not be retained in memory and visual encoding of the new object simply overwrites the original trace. No comparison with the original is possible so the participant is unaware of any change.

This theory predicts that any manipulation that might direct attention toward the changing region should reduce the degree of change blindness experienced by participants. If gaze cues tend to trigger shifts of observers' visual attention, then we might expect a change to an object cued by the direction of someone's gaze to be detected sooner than a change made to an uncued item. This was indeed the case; when the gaze and head orientation of an individual appearing in a scene cued the change made to the display, participants spotted the change over twice as quickly as in scenes where no cue was present or when a neutral cue was provided.

One interpretation of this finding is that gaze cues help a viewer establish some kind of gist or meaning of a scene. The idea is that gaze and head orientation are computed rapidly and trigger attentional shifts within the scene. This, in turn, allows the viewer to build up a representation of the object that is currently the focus of the gazer's attention.

Orienting one's attention on the basis of another's social attention signals also serves as a mechanism for coordinating processing agendas. For instance, Ballard and colleagues [39,40] see gaze as a deictic: a pointer used to mark an object or location for the target of some processing operation. So an individual in a scene foveates an object relevant to their current behavioural goal. Rapid perception of gaze and deployment of the observer's attention ensures that both actor and observer represent the same object. This co-ordination of activity might be particularly important if, as has been suggested by those studying change blindness, detailed representations of objects in scenes only exist for the currently attended item [36].

\section{Gaze in Higher-Level Cognition and Communication}

The studies reviewed in the preceding sections show how gaze cues can influence cognitive activity, even when these signals are sometimes irrelevant to the ongoing task; they trigger reflexive shifts of an observer's attention and produce interference effects on responses to directional words. Ordinarily, of course, gaze cues are delivered in concert with speech and will often serve to augment understanding (e.g., the comprehension of deictic expressions like "this one", or "that one"). Indeed a good deal of research has shown that non-verbal signals have beneficial effects on communication [41, 42]. However, in view of the power cues such as gaze and head orientation have on our cognitive activity we should be mindful of circumstances where they might actually hinder performance in certain higher level cognitive and communciative tasks.

A recent study by Glenberg et al. [43] perhaps illustrates the potential that visual cues delivered by the face might have to disrupt task performance. They reported that when people are asked moderately difficult questions, they often avert their gaze. Moreover, the frequency of gaze aversion was related to the difficulty of cognitive processing, and averting gaze improved task performance. Glenberg et al. suggest that averting gaze helps people to disengage from environmental stimulation and thereby enhances the efficiency of cognitive processing directed by non-environmental stimulation. Recent work here in Stirling suggests that 
children may also engage in the same gaze-averting behaviour as adults. Doherty-Sneddon, et al. [44] have shown that 8- and 9-year old children avert their gaze in response to increasing cognitive difficulty in tasks involving episodic and spatial memory, tests of mental arithmetic and several tasks designed to tap verbal skills. Children and adults therefore seek to avoid potentially distracting facial stimuli when attempting demanding cognitive tasks. Indeed, evidence suggests that compelling children to monitor another's face can significantly impair their performance on a visuo-spatial memory task, and disrupts their ability to decode descriptions of abstract shapes [45]. Similarly disruptive effects of facial signals were noted in a communicative problem solving task where pairs of children described abstract shapes to one another. Here, 6- and 10-year old children performed better in conditions where they were unable to see one another than when they were face-toface [46].

Faces therefore seem to be able to disrupt performance in certain cognitive and communicative tasks. Why should this be so? We have already seen how task-irrelevant gaze and head cues can influence information processing; we might think of these social attention cues as capturing certain processing resources. Moreover, perhaps these resources are the same as those used to perform visuo-spatial memory tasks and to understand descriptions of abstract shapes. The source of the disrupting effect of facial signals might therefore be a competition for specific visuo-spatial processing resources.

\section{Implications}

I now turn to the implications that some of this research might have for the design of robot-human interfaces. Robots have typically been thought of as "appliances", agents designed to perform certain tasks that might be seen as routine or perhaps too dangerous for humans to undertake. Here the challenge for designers is to create a user interface so that untrained users might make efficient use of the robot. Other applications, however, might require a robot to actually engage in social interaction with a human user. For example, a robot "home help" might well be in demand in the near future for our growing elderly population. This raises a rather different challenge to the designer: ensuring that any social exchanges with the robot are natural, understandable, enjoyable, and sufficiently flexible to meet the user's demands. For many, the goal is therefore a "socially intelligent" robot $[47,48]$

Humans have evolved a kind of social intelligence in order to survive in the social world. By this I mean that we have evolved an ability to make sense of another's actions and, crucially, predict what they are about to do next. Our own behaviours are based on what we know, believe or sometimes pretend to be true about the world. So it makes sense to assume that other agents' behaviour is based on the same kinds of mental states. But how do we decide to which agents we should attribute these mental states? We seem happy to conclude that children or chimpanzees act in accordance with their "beliefs", but less inclined to do the same with, say, fishes or insects. The answer is that humans and many non-human primates display social signals which seem to express their internal states. By furnishing it with the ability to deliver these social cues, a robot will at least give the human user the impression that it too has beliefs, desires and intentions that might facilitate social exchange [49]. Given the importance of gaze and head orientation in signalling attention direction, and the use we make of these cues to "read" intentional mental states [28], any socially intelligent robot worth its salt must, at the very least, have eyes and a moving head. Indeed, Breazeal and her colleagues at MIT have developed a robot which uses gaze, as well as posture and facial expression [50].

However, the research described in the preceding sections highlights some possible implications of giving a robot eyes and a moving head. We have seen how gaze and head cues are very difficult to ignore: they produce interference effects on responses to spoken directional words and may capture processing resources that might otherwise be deployed in the performance of certain visuo-spatial tasks. One can therefore imagine applications for which the inclusion of social attention cues might not be appropriate: an on-screen synthetic face, acting as a children's educational tutor, for example. Although research suggests that children and adults disengage from facial stimuli when involved in difficult tasks, it might be that the novel and unusual use of a face might overcome this tendency, exacerbating any interference effects.

Designers might also be aware of the power that gaze and head cues have to trigger shifts of an observer's visual attention and how this might affect the way we represent information in scenes. Certain movements of a robot's eyes and/or head might trigger unwanted shifts, distracting a user from the current focus of attention and causing them to represent and process irrelevant visual information. There are, however, many unanswered questions in this area of research. For example, do all movements of the eyes trigger attentional shifts in an observer, or are the temporal aspects of gaze shifts important here? Recent research has suggested that it is movement per se, rather than movement of the eyes in particular, that triggers infants' shifts of attention [51]. How might head and gaze cues interact in triggering attention shifts? Does one's attention shift if another's head turns but their eyes remain in contact with yours? These are the sorts of things that psychologists and designers might want to research, but also the very questions that robot eyes might help to answer. The movement of a robot's eyes and head can be precisely and independently controlled and readily replicated, making them ideal as stimuli in experiments designed to explore the role that the dynamic aspect of social cues have in social attention.

In addition to being able to send appropriate gaze signals, a socially intelligent robot would also need to be 
able to interpret these same cues. The first challenge for designers here is one of image interpretation: to recover eye-direction from an image of the eye that is not necessarily face-on to the observer. The scleral contrast computation described earlier is one such method. Research has also highlighted the importance of head orientation in social attention; it is an important cue for young children and is processed automatically by observers. Whether it needs to be computed in order to perceive eye-gaze direction is under debate (scleral contrast can signal absolute eye direction in space), but people surely use it as an attentional cue in its own right, and therefore it must be available to a socially intelligent robot. Head orientation therefore also needs to be recovered from the image of the face and recent work has suggested that this can be done by computing the deviation of the head profile from bilateral symmetry and/or the deviation of the nose from vertical [18].

However, once again there are significant challenges for designers and researchers. For example, it is unclear exactly how humans, and therefore robots, are able to disambiguate gaze cues? Shifts of gaze and/or turns of the head serve a number of different functions. They can act as intentionally communicative signals, illustrating the referent of a remark, disambiguating deictic expressions such as "this one" or "that one", expressing intimacy or dominance, or communicating various emotional states. But gaze shifts need not be intentionally communicative at all - for example when we gaze upwards when thinking. Given all the different functions, and the range of meanings the eyes and head might express, it is difficult to imagine how are able, in the most part, to interpret just what another's gaze actually means. One possibility is that different kinds of signals have different spatial and temporal properties which can be used to disambiguate their meanings.

Clearly there is very much more we need to understand about how we process gaze and head orientation, and the effects these cues can have on various aspects of information processing. Continued collaboration between psychologists, neuroscientists and those involved in designing and building socially intelligent agents will be important in this enterprise.

\section{References}

[1] A.W. Young, Face and mind. Oxford: Oxford University Press, 1998.

[2] V. Bruce and A.W. Young, "Understanding face recognition”, Br. J. Psychol., pp. 305-327, Vol. 77, 1986.

[3] H.D. Ellis, "Processes underlying face recognition". In R. Bruyer (Ed.), The neuropsychology of face perception and facial expression, Hillsdale, NJ: Lawrence Erlbaum Associates, 1986.

[4] A.W. Young et al., "Face perception after brain injury: selective impairments affecting identity and expression”, Brain, pp. 941-959, Vol. 116, 1993.
[5] S. Walker, V. Bruce, and C. O'Malley, "Facial identity and facial speech processing: Familiar faces and voices in the McGurk effect", Perception and Psychophysics, pp. 1124 - 1133, vol. 57, 1995.

[6] S.R. Schweinberger, and G.R. Soukup, "Asymmetric relationships among perceptions of face identity, emotion and facial speech", Journal of Experimental Psychology: Human Perception and Performance, pp. 1748 - 1765, vol. 24, 1998.

[7] C.L. Kleinke, "Gaze and eye contact: A research review", Psychological Review, pp. 78-100, vol. 100, 1986.

[8] J.J. Gibson and A. Pick, A. "Perception of another person's looking", American Journal of Psychology, pp. 86-94, vol. 76, 1963.

[9] M.G. Cline, "The perception of where a person is looking", American Journal of Psychology, pp. 4150, vol. 80, 1967.

[10] R.J. Watt, "What your eyes tell my eyes, and how your eyebrows try to stop them" Paper presented at the Tenth International Conference on Perception and Action, University of Edinburgh, August, 1999.

[11] C. Lord and M.M. Haith, "The perception of eye contact", Perception and Psychophysics, pp. 413416, vol. 16, 1974.

[12] S.R.H. Langton, R.J. Watt and V. Bruce, "Do the eyes have it: Cues to the direction of social attention", Trends in Cognitive Science, pp. 50-58, vol. 4, 2000.

[13] D.I. Perrett, J.K Hietanen, M. W. Oram, and P.J. Benson, "Organisation and functions of cells responsive to faces in the temporal cortex". Philosophical Transactions of the Royal Society of London, Series B, pp. 23-30, vol. 335, 1992.

[14] D.I. Perrett, P.A.J. Smith, D.D. Potter, A.J. Mistlin, A.S. Head, A.D. Milner, and M.A. Jeeves, "Visual cells in the temporal cortex sensitive to face view and gaze direction", Proceedings of the Royal Society of London, pp. 293-317, , vol. B223, 1985.

[15] C.A. Heywood and A. Cowey, "The role of the 'face cell' area in the discrimination and recognition of faces by monkeys", Philosophical Transactions of the Royal Society of London, Series B., pp. 31-38, vol. 335, 1992.

[16] R. Campbell, C.A. Heywood, A. Cowey, M. Regard and T. Landis, "Sensitivity to eye gaze in prosopagnosic patients and monkeys with superior temporal sulcus ablation", Neuropsychologia, pp. 1123-1142, vol. 28, 1990.

[17] J.V. Haxby, E.A. Hoffman and M.I. Gobbini, "The distributed human neural system for face perception", Trends in Cognitive Sciences, pp. 223233, vol. 4, 2000.

[18] H.R. Wilson, F. Wilkinson, L-M Lin and M Castillo, "Perception of head orientation", Vision Research, pp. 459-472, vol. 40, 2000.

[19] V. Corkum, and C. Moore, "Development of joint visual attention in infants", In C. Moore and P.J Dunham (Eds.) Joint attention: Its origins and role 
in development, pp. 61-83, Lawrence Erlbaum Associates, 1995

[20] D.I. Perrett and N.J. Emery, N. J, "Understanding the intentions of others from visual signals: Neurophysiological evidence", Cahiers de Psychologie Cognitive, pp. 683-694, vol. 13, 1994.

[21] J. Fodor, The modularity of mind: An essay on faculty psychology. Cambridge, MA: MIT Press, 1983.

[22] S.R.H. Langton, "The mutual influence of gaze and head orientation in the analysis of social attention direction" Quarterly Journal of Experimental Psychology, pp. 825-845, vol. 53, 2000.

[23] S.R.H. Langton, and V. Bruce "You Must See the Point: Automatic Processing of Cues to the Direction of Social Attention", Journal of Experimental Psychology: Human Perception and Performance, pp. 747-757, vol. 26, 2000.

[24] S.R.H. Langton, C. O’Malley and V. Bruce, "Actions speak no louder than words: Symmetrical cross-modal interference effects in the processing of verbal and gestural information", Journal of Experimental Psychology: Human Perception and Performance, pp. 1357-1375, vol. 22, 1996.

[25] M. Scaife and J.S. Bruner, "The capacity for joint visual attention in the infant", Nature, pp. 265-266, vol. $253,1975$.

[26] G. Butterworth and N. Jarrett, N, "What minds have in common is space: Spatial mechansims serving joint visual attention in infancy" British Journal of Developmental Psychology, pp. 55-72, vol. 9, 1991.

[27] C. Moore and V. Corkum, "Infant gaze following based on eye direction" British Journal of Psychology, pp. 495-503, vol. 16, 1998.

[28] S. Baron-Cohen, "How to build a baby that can read minds: Cognitive mechanisms in mindreading", Cahiers de Psychologie Cognitive, pp. 513-552, vol. 13, 1994.

[29] S.R.H. Langton, and V. Bruce, "Reflexive visual orienting in response to the social attention of others" Visual Cognition, pp. 541-567, vol. 6, 1999.

[30] C.K. Friesen and A. Kingstone, "The eyes have it!: reflexive orienting is triggered by nonpredictive gaze", Psychonomic Bulletin and Review, pp. 490495, , vol. 5, 1998.

[31] J. Driver, G. Davis, P. Ricciardelli, P. Kidd, E. Maxwell and S. Baron-Cohen, "Shared attention and the social brain: Gaze perception triggers automatic visuospatial orienting in adults", Visual Cognition, pp. 509-540, vol. 6, 1999.

[32] M.I. Posner, "Orienting of attention". Quarterly Journal of Experimental Psychology, pp. 3-25, vol. 32, 1980.

[33] S.R.H. Langton and C. O'Donnell, "Gaze cues attenuate change blindness in the flicker paradigm" (manuscript in preparation).

[34] R.A. Rensink, J.K. O'Regan and J.J. Clark, "To see or not to see: The need for attention to perceive changes in scenes", Psychological Science, pp. 368373, vol. 8, 1997.

[35] D.J. Simons and D.T. Levin, "Change blindness", Trends in Cognitive Sciences, pp. 261-267, vol. 1, 1997.

[36] R.A. Rensink, "The dynamic representation of scenes", Visual Cognition, pp. 17-42, vol. 7, 2000.

[37] B.J. Scholl, "Attenuated change blindness for exogenously attended items in a flicker paradigm", Visual Cognition, pp. 377-396, vol. 7, 2000.

[38] K.L. Shapiro, "Change blindness: Theory or paradigm?", Visual Cognition, pp. 83-92, vol. 7, 2000.

[39] D.H. Ballard, M. Hayhoe, P. Pook and R. Rao, "Deictic codes for the embodiment of cognition", Behavioral and Brain Sciences, 723-767, vol. 20, 1998.

[40] M. Hayhoe, "Vision using routines: A functional account of vision", Visual Cognition, pp. 43-64, vol. 7, 2000.

[41] E.A. Boyle, A.H. Anderson and A. Newlands, "The effects of visibility on dialogue and performance in a cooperative problem solving task", Language and Speech, pp. 1-20, vol. 37, 1994.

[42] G. Doherty-Sneddon, A. Anderson, C. O’Malley, S. Langton, S. Garrod and V. Bruce, V. "Face-to-face and video-mediated communication: A comparison of dialogue structure and task performance", Journal of Experimental Psychology: Applied, pp. 105-127, vol. 3, 1997.

[43] A.M. Glenberg, J.L. Schroeder, D.A. Robertson "Averting the gaze disengages the environment and facilitates remembering" Memory \& Cognition, pp. 651-658, vol. 26, 1998.

[44] G. Doherty-Sneddon, L. Bonner and C. Doyle, "Development of gaze aversion as disengagement from visual information", Manuscript submitted to British Journal of Developmental Psychology.

[45] G. Doherty-Sneddon, V. Bruce and L. Bonner, "Cognitive demands of face processing", Memory and Cognition, in press.

[46] G. Doherty-Sneddon, S. McAuley, V. Bruce, S. Langton, A. Blokland and A. H. Anderson, "Visual signals and children's communication: Negative effects on task outcome", British Journal of Developmental Psychology, pp. 595-608, vol. 18, 2000.

[47] B. Adams, C. Breazeal, R.A. Brooks and B. Scassellati, "Humanoid robots: A new kind of tool", IEEE Intelligent Systems, August, 2000.

[48] B. Scassellati. "Investingating Models of Social Development Using a Humanoid Robot," in B. Webb and T. Consi, (eds)., Biorobotics, Cambridge, MA: MIT Press, in press.

[49] C. Breazeal and B. Scassellati, "How to build robots that make friends and influence people" Proceedings of the International Conference on Intelligent Robots and Systems, Kyongju, Korea, 1999. 
[50] C. Breazeal, A. Edsinger, P. Fitzpatrick, B. Scassellati, P. Varchavskaia, "Social constraints on animate vision", IEEE Intelligent Systems, August, 2000

[51] T. Farroni, M.H. Johnson, M. Brockbank and F. Simion, "infants' use of gaze direction to cue attention: The importance of perceived motion", Visual Cognition, pp. 705-718, vol. 7, 2000. 